\title{
El patrimonio industrial convertido en museo: el caso del IAACC Pablo Serrano de Zaragoza
}

\author{
ELENA MARCÉN GUILLÉN \\ Becaria de Investigación. Departamento de Historia del Arte / Universidad de Zaragoza \\ Miembro del Grupo de Investigación Observatorio Aragonés de Arte en la Esfera Pública \\ emarcen@unizar.es
}

\begin{abstract}
Resumen
La reconversión del patrimonio industrial para usos museísticos se ha revelado como un fenómeno de creciente importancia en las últimas décadas. Este afán de reutilización es debido (entre otras causas) a las posibilidades físicas que ofrece la arquitectura industrial para la instalación de museos; pero también a la voluntad de los arquitectos contemporáneos por dejar su impronta en la ciudad. Los ejemplos son variados; en función de la mayor o menor fortuna a la hora de integrar la arquitectura contemporánea y la histórica. En la presente comunicación planteamos el estudio monográfico de un ejemplo zaragozano de arquitectura industrial rehabilitada para usos museísticos: el Instituto Aragonés de Arte y Cultura Contemporáneos Pablo Serrano.

El Museo Fundación Pablo Serrano abrió sus puertas en 1994 en los antiguos talleres del Hogar Pignatelli; construidos a principios del siglo XX según diseño del arquitecto Julio Bravo y reconvertidos en museo entre 1987 y 1993. El arquitecto encargado de esta remodelación, José Manuel Pérez Latorre, fue designado también para ocuparse de la reciente ampliación del museo, llevada a cabo a partir de 2008 y que ha dado como resultado un nuevo espacio enfocado a la cultura y el arte contemporáneos. Esta última intervención arquitectónica, muy cuestionada por su volumetría y aspecto externo, resulta de gran interés para analizar las relaciones que establece el museo con la ciudad. El IAACC Pablo Serrano se inserta en una zona de tejido urbano heterogéneo, que combina una serie de edificios representativos con bloques de viviendas sin personalidad. En la presente comunicación analizaremos el diálogo (fluido o no) que entabla el museo con su entorno, tratando de discernir los aciertos y errores de una de las propuestas culturales "estrella" del anterior gobierno autonómico.
\end{abstract}

Palabras clave: Arquitectura industrial; arquitectura de museos; Zaragoza; IAACC; Pablo Serrano; José Manuel Pérez Latorre. 


\title{
The industrial heritage restored as a museum: the IAACC Pablo Serrano case in Zaragoza
}

\begin{abstract}
The restoration of the industrial heritage for its use as museums has been revealed as an increasingly important phenomenon in the past few decades. This pursuit of reuse is due (among other causes) to the physical possibilities that industrial architecture offers for establishing new museums, but also to the willingness of contemporary architects for leaving their mark on the city. There are various examples according to the grade of success when integrating both modern and historical architectures. This paper is a monographic study case of a great example of industrial architecture turned to museum: The IAACC Pablo Serrano in Zaragoza (Spain).

The Museum-Foundation Pablo Serrano opened its doors in 1994 in the old workshops of the Hogar Pignatelli; built in the early 20th century as planned by the architect Julio Bravo and converted into museum between 1987 and 1993. The architect in charge of this remodeling, José Manuel Pérez Latorre, was also designated as head of the latest enlargement of the museum in 2008, which has resulted in a brand new space focused to contemporary art and culture. This latest intervention -sometimes questioned because of its bulkiness and exterior- is of huge interest in order to analyze the relationships established between the museum and the city. The IAACC Pablo Serrano fits into an area of heterogeneous urban fabric, which combines a series of representative buildings alongside residential blocks without personality. In the present communication the dialog (either fluid or not) the museum creates with its environment is analyzed, evaluating the rights and wrongs of one of the flagship cultural proposals of the previous regional government.
\end{abstract}

Key words: Industrial architecture; museum architecture; Zaragoza; IAACC; Pablo Serrano; José Manuel Pérez Latorre.

Sumario: 1.- Introducción: el éxito de la recuperación del patrimonio industrial para su reconversión en museo. 2.- La sede del IAACC Pablo Serrano: historia de los talleres del Hogar Pignatelli. 2.1.- Creación y trayectoria de la Fundación Museo Pablo Serrano 2.2.- De talleres a museo. Arquitectura de la primera etapa del Museo Pablo Serrano (1994-2007) 2.3.- La influencia del contexto urbano en las nuevas formas del museo 2.4.- Nuevas formas para un nuevo museo: la ampliación del IAACC Pablo Serrano 3.- Conclusiones. 4.- Referencias bibliográficas. 


\section{Introducción: el éxito de la recuperación del patrimonio industrial para su reconversión en museo.}

La reconversión del patrimonio industrial para usos museísticos se ha revelado como un fenómeno de creciente importancia en las últimas décadas. Este afán de reutilización es debido (entre otras causas) a las posibilidades físicas que ofrece la arquitectura industrial para la instalación de museos, pero también a la voluntad de los arquitectos contemporáneos por dejar su impronta en las ciudades. Los ejemplos son variados, en función de la mayor o menor fortuna a la hora de integrar la arquitectura contemporánea y la histórica. Uno de los casos ya paradigmáticos es la rehabilitación de la antigua central eléctrica de Bankside en Londres llevada a cabo por los suizos Herzog \& De Meuron para albergar la Tate Modern. Los mismos arquitectos se encargarían también de la rehabilitación de otra central eléctrica para usos museísticos, esta vez más cerca: el CaixaForum de Madrid en la antigua central eléctrica del Mediodía.

Ascensión Hernández (2007: 32-35) distingue dos tendencias extremas en cuanto a la recuperación de infraestructuras industriales para usos culturales: aquellas intervenciones más radicales en las que la arquitectura actual se impone sin consideración alguna sobre la histórica, (...) por la ruptura de escala de la parte nueva respecto a la arquitectura histórica y el entorno urbano circundante, y otras más respetuosas con la fábrica original, en las que los arquitectos contemporáneos han respetado conscientemente las características tipológicas, formales y materiales de la arquitectura industrial preexistente, (...) utilizando la arquitectura nueva como un recurso para potenciar los valores del edificio restaurado.

En la presente comunicación planteamos el estudio monográfico de un ejemplo zaragozano que cabría clasificar dentro de esa primera tendencia más radical en la rehabilitación de arquitectura industrial para usos museísticos: el Instituto Aragonés de Arte y Cultura Contemporáneos Pablo Serrano.

\section{La sede del IAACC Pablo Serrano: historia de los talleres del Hogar Pignatelli.}

El IAACC Pablo Serrano está ubicado en los antiguos talleres de la institución asistencial conocida como Casa de Misericordia u Hogar Pignatelli de Zaragoza. Estos talleres fueron creados en el siglo XVIII como medio de recaudar fondos para el hogar, aunque tuvieron también una importante función como centro de formación profesional. En un principio los talleres esta- 
ban ubicados dentro del recinto del Hogar, aunque a comienzos del siglo XX se vio la necesidad de dotarlos de un espacio propio para ampliar y modernizar sus instalaciones, que se hará realidad con el desarrollo del proyecto presentado en 1910 por el arquitecto provincial Julio Bravo.

El conjunto estaba formado por dos bloques unidos: el de los talleres propiamente dichos, que toma como modelo el tipo de edificio industrial de tres naves paralelas con cubierta en dientes de sierra orientadas hacia el norte, que acogían los talleres de herrería, hojalatería y carpintería; y un bloque de dos alturas dispuesto de forma perpendicular a las naves anteriores que albergaba el Pabellón de Música y Pintura. La configuración formal de los dos volúmenes era distinta, de tal manera que los talleres mostraban una fachada principal de gran simplificación formal, mientras que el bloque perpendicular presentaba una mayor riqueza compositiva.

Tras la pérdida de función de los talleres en la década de los años 40 del siglo XX, estos quedaron en desuso y llegaron a los años 80 en un estado de abandono generalizado.

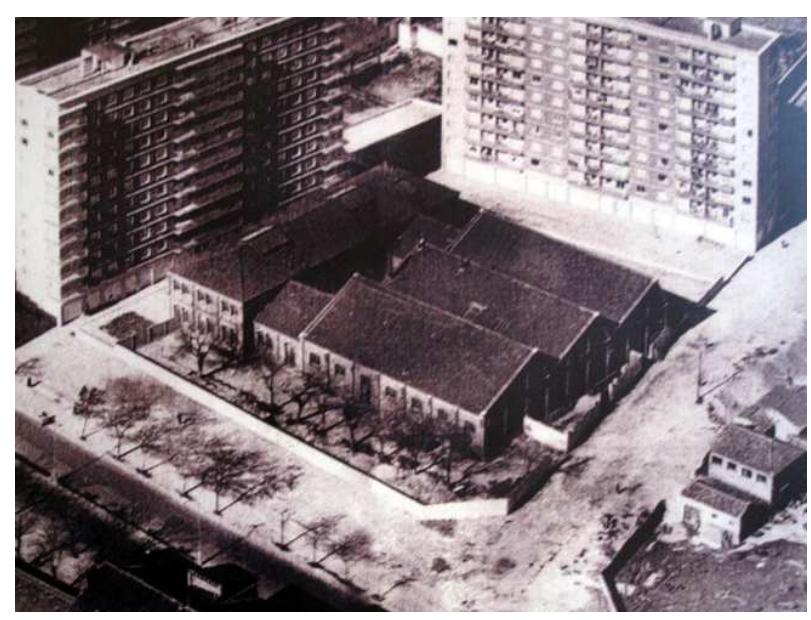

Fig. 1. Talleres del Hogar Pignatelli antes de su reconversión en Museo Pablo Serrano.

\subsection{Creación y trayectoria de la Fundación Museo Pablo Serrano.}

El IAACC Pablo Serrano tiene su origen en la voluntad del escultor de Crivillén de que su obra permaneciera en Aragón a su muerte. Los contactos entre el escultor y las administraciones dieron sus frutos en la constitución, el 29 de julio de 1985, de la Fundación Museo Pablo Serrano como fundación 
cultural privada ${ }^{1}$. En ella estaban presentes todas las instituciones oficiales aragonesas, así como relevantes personas del mundo de la cultura. El fin primordial de la Fundación era la creación de un museo con las obras donadas por el escultor, aunque también se pretendía promover la formación, investigación y difusión artística en sus múltiples vertientes. Ese mismo año, y tras barajar posibles ubicaciones, se decidió la reutilización de los edificios de los antiguos talleres que eran entonces propiedad de la Diputación Provincial de Zaragoza (y que la institución cederá en 1987) para situar en ellos el museo. Serrano murió unos meses más tarde, en noviembre de 1985.

El día 2 de junio de 1986 se celebró en Madrid la firma de la escritura de acuerdo entre los herederos del escultor y la Fundación para la donación de las primeras obras del artista, lo que suponía la finalización del proceso legal de puesta en marcha del proyecto. Con las primeras obras que llegaron a Zaragoza se realizó una muestra antológica en el palacio de la Lonja.

El Museo Pablo Serrano no abrió sus puertas hasta el 27 de mayo de 1994, casi diez años después de la constitución de la Fundación, tras haber acumulado constantes retrasos motivados por la falta de financiación, que hicieron que el coste del museo se triplicara desde lo previsto en un principio. Sin embargo, la historia de la institución continuó siendo azarosa: un año después de la apertura del museo, la Fundación se extinguía por la imposibilidad de realizar su fin fundacional, pasando la propiedad de sus bienes y derechos a la Comunidad Autónoma de Aragón.

Tras la disolución de la Fundación se creaba el Instituto Aragonés del Arte y la Cultura Contemporáneos Pablo Serrano ${ }^{2}$, que supuso un cambio de modelo al convertirse en el eje de las políticas culturales en materia de arte contemporáneo del Gobierno de Aragón. El nuevo Instituto, que venía a sustituir al Museo Aragonés de Arte Contemporáneo creado por decreto en 1993 y que nunca llegó a ser una realidad, reunía así en sus fondos la colección que ya

\footnotetext{
${ }^{1}$ La constitución de la Fundación tuvo eco en la prensa nacional: J. Ortega: “Constituida en Zaragoza la Fundación Museo Pablo Serrano", El País, (Madrid, 31-VII-1985).

${ }^{2}$ Decreto 218/1995, de 5 de julio, de la Diputación General de Aragón, por el que se crea el Instituto Aragonés del Arte y la Cultura Contemporáneos “Pablo Serrano"), BOA no 100, de 18 de agosto de 1995.
} 
era propiedad de la Diputación General de Aragón y las obras procedentes de la Fundación-Museo Pablo Serrano. Es así como el centro, inicialmente concebido como museo monográfico, fue asumiendo de manera progresiva una actividad más variada.

\subsection{De talleres a museo. Arquitectura de la primera etapa del museo Pablo Serrano (1994-2007).}

La rehabilitación y adaptación de los antiguos talleres para usos museísticos fue llevada a cabo por el arquitecto zaragozano José Manuel Pérez Latorre. Este primer proyecto de adecuación tuvo como resultado en líneas generales un museo respetuoso con el edificio industrial original; el propio Pérez Latorre reconocía que el edificio original domina sobre la idea, ya que esta ha de surgir de lo ya existente (Pérez Latorre, 1990: 2).

La solución obtenida vino dada en parte por las propias características físicas del espacio industrial. Las tres naves paralelas que acogían los talleres se encontraban separadas por muros, que Pérez Latorre eliminó para conseguir un único espacio apto para acoger la sala principal de la exposición. Desde el punto de vista estructural, la obtención de un gran y único espacio fue posible gracias a la colocación de tres grandes cerchas que permitían redistribuir los pesos de las cubiertas a una serie de pilares de hormigón armado. Aunque se eliminó la compartimentación interior, la división en tres naves seguía siendo perceptible en altura.

La luz se convertía en protagonista indiscutible del proyecto. Este elemento condicionaba no solo la sala principal de exposición sino también el exterior del edificio, para cuya fachada se escogió crear una pantalla de hormigón armado situada ante la delantera original de los talleres, creando un espacio de tránsito entre ambas. La configuración volumétrica de esta fachada, en combinación con la incidencia de la luz, creaba un juego de luces y sombras que otorgaba un ritmo constante a la fachada. A ello había que añadir la entrada concebida por el arquitecto, situada al otro lado de un ligero puente sobre un foso de agua como estructura de reminiscencias venecianas. La inserción de esta lámina acuática conseguía suavizar el hormigón de la fachada y otorgaba al museo una consideración metafórica de oasis dentro de la ciudad. 
Fig. 2.

Museo Pablo Serrano.

Fuente: Fotografía de José Manuel Pérez Latorre.

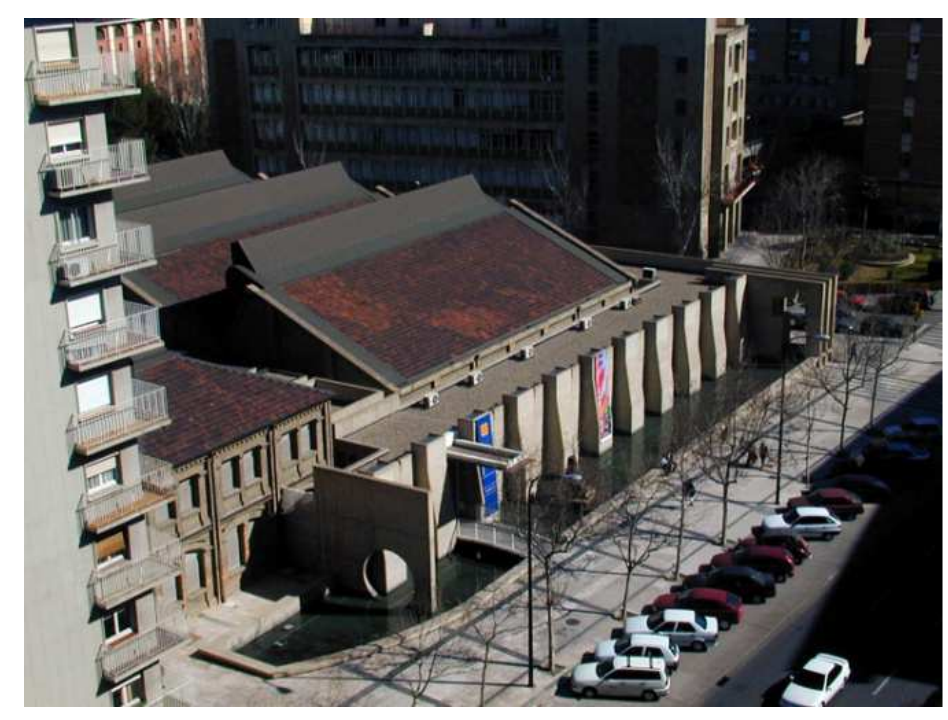

Uno de los aspectos significativos del proyecto es que el mismo arquitecto se ocupó de la museografía, con el diseño de la distribución de la sala principal de exposición, de la distribución de la colección de Pablo Serrano y de los sistemas expositivos. Pérez Latorre planteó una organización descendente, en una espiral en varios niveles a los que se iba accediendo mediante rampas, una estructura que recordaba al museo de crecimiento ilimitado de Le Corbusier. Se concebían dos recorridos en función del tipo de visitante: uno para escolares, más breve y general, que estaría situado en las paredes de la sala y los muros perimetrales de los distintos desniveles, y otro para adultos, más completo y que incluiría las zonas diferenciadas por los desniveles.

Los muros perimetrales y las vitrinas se convertían en una pieza escultórica más, alcanzando quizá un protagonismo excesivo, pero lo cierto es que el resultado era una sala de exposición original, de proporciones coherentes y que permitía una visión general de la obra de Serrano. La dedicación inicial de las naves estaba presente en las cubiertas en dientes de sierra que, aunque transformadas, seguían hablando de su concepción arquitectónica como talleres.

El Museo Pablo Serrano cerró sus puertas en julio de 2007 para acometer las obras de ampliación, necesarias para aumentar la superficie disponible. 

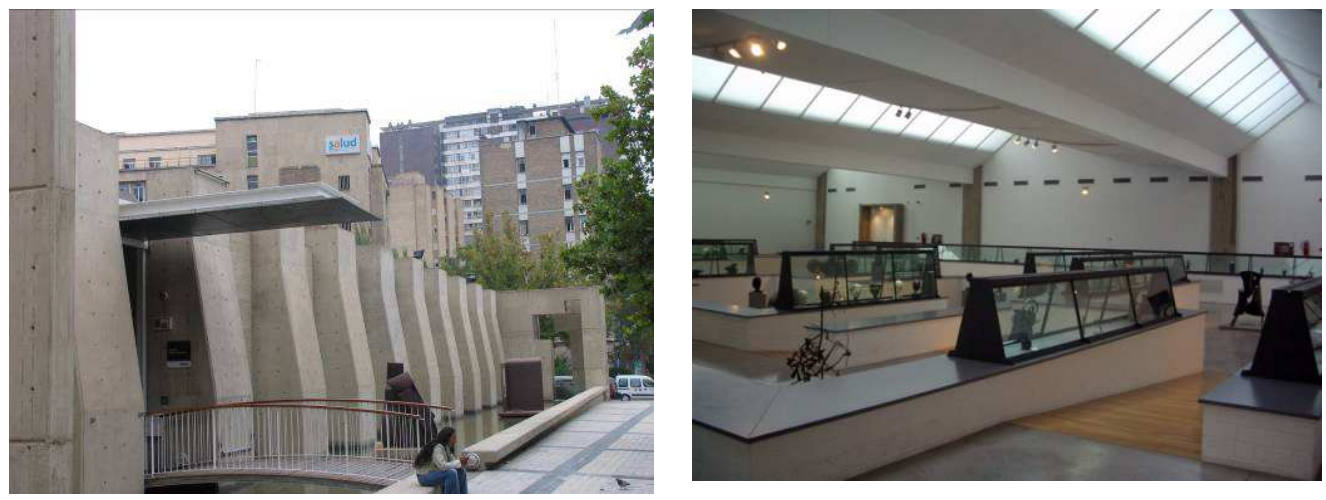

Figs. 3 y 4. Fachada y Sala de exposición permanente del Museo Pablo Serrano Fuente: Fotografías de José Manuel Pérez Latorre

\subsection{La influencia del contexto urbano en las nuevas formas del museo.}

Antes de abordar de forma monográfica la ampliación del IAACC Pablo Serrano resulta interesante presentar, en unas pocas pinceladas, la trama urbana en la que se enmarca. Este entorno urbano, que es el resultado de la suma de piezas aisladas desde el punto de vista arquitectónico, tiene su origen en la urbanización de las huertas y terrenos de distintos conventos e instituciones que resultaron muy afectados durante los Sitios de Zaragoza (1808-1809), y que fueron urbanizados progresivamente hasta crear una trama urbana más o menos compacta. En este contexto han ido surgiendo a lo largo del tiempo una serie de edificios representativos tanto civiles como religiosos que conviven con bloques de viviendas de entre cinco y diez alturas, configurando así un complejo entorno urbano cuyas características principales resumiremos a continuación.

El IAACC Pablo Serrano se encuentra localizado en el paseo de María Agustín № 20, una importante arteria ciudadana que coincide con la ubicación de la muralla de época medieval de la ciudad y que hoy constituye el segundo cinturón de Zaragoza, una de las vías de acceso al centro urbano. En este paseo podemos distinguir dos zonas: la acera de los impares, con un tejido urbano esencialmente residencial, y la acera de los pares, marcada por la presencia de edificios singulares de uso institucional y religioso, aunque también hay algunos bloques de viviendas. Esta acera de los pares responde a una urbanización tardía, efectuada en la década de los años 60 del siglo XX, lo que explica que se realizara sin solución de continuidad, a partir de edificios aislados y descontextualizados. 
Uno de los monumentos más representativos de este ámbito ciudadano es la Puerta del Carmen, situada en la confluencia de la avenida de César Augusto, los paseos de Pamplona y María Agustín y la calle de Hernán Cortés. La puerta, datada a finales del siglo XVIII y cuyo diseño se ha atribuido tradicionalmente a Agustín Sanz $\mathrm{z}^{3}$, se muestra hoy desprovista de su misión original de entrada a la ciudad y ha quedado aislada, con una función meramente ornamental.

Muy próximo a la Puerta del Carmen se sitúa el monumental convento de las Madres Carmelitas de la Encarnación, construido entre 1962 y 1965 según proyecto de Eduardo Lagunilla sobre el lugar en el que se encontraba el antiguo desde el siglo XVII. La ubicación del edificio hubo de modificarse (retrocediendo varios metros) según una ordenación urbanística llevada a cabo por el Ayuntamiento para crear la avenida de César Augusto. Siguiendo esa misma acera de los pares, que nos conduce al IAACC Pablo Serrano, encontramos el edificio Ebrosa (conocido así por la constructora que lo realizó), construido a finales de la década de los años sesenta y que marcaría el camino para otras construcciones monumentalistas. A continuación se sitúa la iglesia de Nuestra Señora del Carmen, de José Romero Aguirre, una pieza arquitectónica de gran modernidad construida entre 1963 y 1965 y compuesta por dos bloques, uno horizontal que corresponde con la nave de la iglesia y otro vertical, situado a la izquierda, en el que se ubica la residencia y cuyo volumen sirve de transición con el edificio vecino. Destaca como elemento compositivo la presencia de la aguja que se eleva en altura en forma de homenaje a la tradicional torre.

Continuando este paseo ciudadano encontramos la antigua Delegación del Ministerio de Trabajo, una obra construida en 1962 por Fernando García Mercadal (autor también del vecino ambulatorio de Ramón y Cajal, hoy Centro de Especialidades) que se sitúa en la calle Doctor Fleming, justo enfrente del IAACC Pablo Serrano. Pasado el museo hay una serie de bloques de antiguas viviendas para empleados de la Diputación Provincial de Zaragoza, tres de ellos dispuestos de forma perpendicular al trazado del paseo y un cuarto paralelo al mismo, así como un solar sin construir. Seguidamente encontramos

${ }^{3}$ Esta autoría ha sido recientemente puesta en duda por Javier Martínez Molina (2009). 
la Jefatura Superior de Policía y el Edificio Pignatelli o Casa de la Misericordia (actual sede del Gobierno de Aragón), a la que pertenecían en origen los talleres Pignatelli.

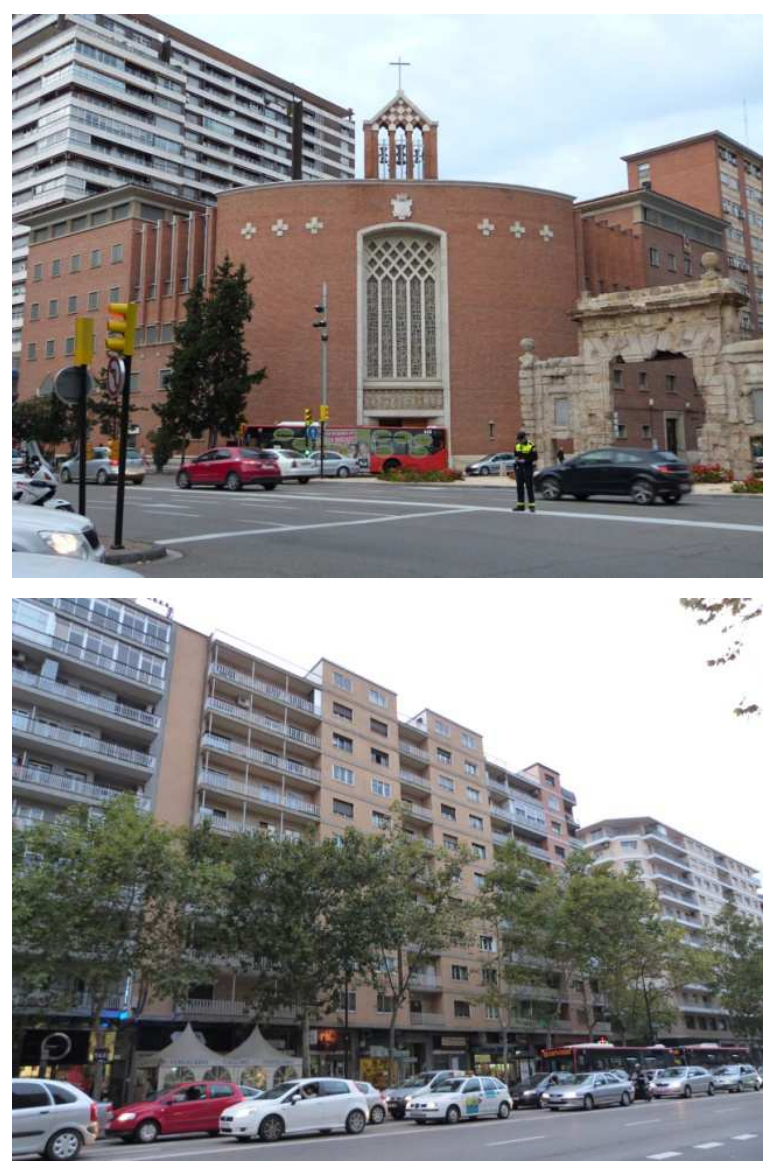

Fig. 5. Puerta del Carmen, Convento de la Encarnación y edificio Ebrosa.

Fig. 6. Paseo de María Agustín (acera de los impares)

Cruzando a la acera de los impares podemos contemplar el grupo escolar Joaquín Costa, diseñado por Miguel Ángel Navarro, un conjunto de 1929 que presenta un aspecto exterior institucional y académico de reminiscencias neoclásicas. Esta acera presenta una urbanización más continua, formada por bloques de viviendas de cinco pisos que conviven con otros de seis, nueve e incluso diez alturas. Algunos de ellos presentan cierto interés, como el situado en el $\mathrm{n}^{\mathrm{o}} 7$ del paseo, un bloque racionalista proyectado por el arquitecto José Beltrán Navarro. 
El resultado final de esta operación de urbanización es, más que un tejido urbano continuo, un collage compuesto por piezas singulares que surgen como entidades aisladas del contexto en el que se insertan. Es una zona compleja desde el punto de vista arquitectónico y poco amable para el ciudadano por su gran volumen de tráfico rodado.

José Manuel Pérez Latorre, arquitecto escogido para la ampliación del museo, es consciente de la dificultad de este entorno urbano. En la memoria justificativa del proyecto arquitectónico (Pérez Latorre, 2006: 6) se lee que este fragmento de ciudad (...) muestra constantemente una voluntad de edificios singulares, que vendría dada según el arquitecto por la estructura de propiedad del suelo a la que hemos aludido con anterioridad. A la vez, Pérez Latorre manifiesta su pretensión de que la ampliación convierta al museo en un nuevo foco de atracción en este entorno urbano:

En los estudios previos hicimos un alzado de toda la calle, y desde la Puerta del Carmen hasta el Pignatelli hay numerosos edificios especiales, edificios construidos en sí mismos, con personalidad propia. El Museo Serrano se va a sumar a ellos ${ }^{4}$.

En este complejo entorno trufado de hitos aislados, la solución arquitectónica era completamente abierta y quizá por ello de gran dificultad. En este sentido la propuesta de Pérez Latorre resulta impactante por la volumetría de sus formas y ajena a un contexto que no sirve de inspiración, debido a su complejidad y carácter inconexo.

\subsection{Nuevas formas para un nuevo museo: la ampliación del IAACC Pablo Serrano.}

Como hemos avanzado, el arquitecto escogido para hacerse cargo de la ampliación del museo fue el mismo José Manuel Pérez Latorre, a quien fue adjudicado el contrato de consultoría y asistencia el 13 de mayo de 2005. Se desconoce el procedimiento seguido para la elección; de hecho, hubo quienes cuestionaron la reelección de este arquitecto, llegando a sugerir que se le

\footnotetext{
${ }^{4}$ M. García: "Pérez Latorre: 'Este es el edificio en el que me he sentido más libre en mi trabajo'", Heraldo de Aragón, Suplemento especial "El nuevo Pablo Serrano. Un gran espacio para el arte", (Zaragoza, 23-III-2011).
} 
había adjudicado el proyecto "a dedo" (el Gobierno de Aragón) siempre negó.

Durante el año 2006 se suspendieron todas las actividades complementarias del museo para iniciar las tareas de acondicionamiento de los fondos, en previsión del comienzo de las obras de ampliación. La exposición permanente de Pablo Serrano siguió mostrándose ese año, y el resto de fondos fueron almacenados en la sala de exposiciones temporales 3B, que había sido acondicionada como sala de reserva, hasta que más adelante fueron llevados a Madrid. Tras el traslado de las oficinas a una sede temporal, el museo cerró sus puertas el 2 de julio de 2007.

La necesidad de limitarse a la superficie ya construida determinó la ampliación del museo en altura, según lo dispuesto en el Plan General de Ordenación Urbana de Zaragoza. Otro de los condicionantes era la necesidad de conservar la fábrica anterior: el edificio de Julio Bravo estaba protegido en su conjunto aunque se permitían obras de rehabilitación, debiéndose conservar en todo momento las fachadas, que debían ser siempre visibles.

En cuanto al sistema de construcción, el edificio se levanta sobre cuatro grandes pilares de hormigón de 2,2 $\mathrm{m}$ de diámetro en su base, que soportan 1.900 toneladas, lo que permite obtener plantas diáfanas con múltiples posibilidades expositivas y dejar los muros de los talleres de Julio Bravo exentos de cargas. La intervención en las antiguas naves supuso abrir en cubierta los huecos necesarios para el paso de los grandes soportes de hormigón armado y desmontar las cerchas curvas colocadas en la primera fase del museo. Las obras sufrieron una serie de problemas estructurales que causaron retrasos: hubo que recalcular las sobrecargas del edificio e introducir cambios en cuanto a la climatización para adaptarse a las normas internacionales.

\footnotetext{
${ }^{5}$ El grupo parlamentario del Partido Popular presentó el 2 de marzo de 2011 una moción en la Comisión de Educación, Cultura y Deporte de las Cortes de Aragón para que se encargara la realización de una auditoría externa sobre el proyecto de ampliación del museo. En rueda de prensa, el diputado popular Miguel Navarro decía que la adjudicación del proyecto arquitectónico se había producido "a dedo". Fuente: Europa Press, "El PP solicita una auditoría sobre el proyecto de ampliación del Pablo Serrano", en Heraldo de Aragón, (Zaragoza, 1-III-2011).
} 
La ampliación triplicó el espacio útil del centro, cuyas superficies útiles pasaron a ser de 7.479, $96 \mathrm{~m}^{2}$. La excavación en profundidad de la zona antes dedicada a la exposición permanente permitió disponer de una amplia planta sótano con archivos, almacenes y talleres de restauración. En la planta baja, además de los espacios de acogida de visitantes, tienda y cafetería (que aún no está en marcha), se ha creado una sala de exposiciones temporales que coincide aproximadamente con la permanente de la anterior etapa. Esta planta se completa con una sala de conferencias, biblioteca, sala de recepciones y espacios pedagógicos. Las plantas $1^{\underline{a}}$ y $2^{2}$ acogen los ámbitos dedicados a la exposición de la obra de Pablo Serrano, así como espacios administrativos, mientras que las plantas $3^{\underline{a}}$ y $4^{\underline{a}}$ están ocupadas casi íntegramente por salas de exposición de carácter diáfano y gran amplitud. La planta $5^{\underline{a}}$, finalmente, ofrece una terraza con excepcionales vistas de la ciudad. La museografía del centro ha sido realizada por Moreno \& Asociados y presenta un carácter industrial, casi provisional, acorde con las nuevas tendencias en arte contemporáneo y con la propia idiosincrasia del edificio.

Las características iniciales del museo (horizontalidad y neutralidad) han sido totalmente modificadas en la ampliación. El museo se configura como un volumen rotundo, con entrantes y salientes, que combina zonas acristaladas con un revestimiento en negro y en turquesa, este último reservado al volumen lateral de comunicación y que evocaría -según el propio arquitecto-el intenso azul de los pantanos del Pirineo ${ }^{6}$. El revestimiento es de Alpolic (fabricado por Mitsubishi Plastics) y busca conseguir el reflejo de los cambios lumínicos ambientales. El nuevo museo trataría de distinguirse del carácter doméstico de los edificios circundantes; de esta voluntad de independencia formal vendría, según se refleja en la memoria, lo abstracto de las formas elegidas (Pérez Latorre, 2006: 7).

El edificio presenta dos fachadas principales: la que da a la calle Doctor Fleming, que es la que el visitante percibe cuando se dirige al museo desde la Puerta del Carmen, y la que abre al paseo de María Agustín, cuya visión completa solo se obtiene desde la otra acera del paseo. El propio arquitecto

${ }^{6}$ C.P.B., “Un edificio que no deja indiferente a nadie”, Heraldo de Aragón, (Zaragoza, 24-VII-2010). 
señalaba en el transcurso de las obras que había dedicado una atención especial a esa fachada lateral en escorzo como carta de presentación del nuevo museo $^{7}$. Esta fachada lateral es la mejor resuelta del edificio, ya que el color turquesa tiene la virtud de dulcificar el volumen, algo que no ocurre en otras perspectivas del mismo. El edificio se concibe de forma externa como una gran pieza escultórica, algo claramente perceptible en la enorme cantidad de bocetos, acuarelas, dibujos, modelados y maquetas que formaron parte del proceso creativo ${ }^{8}$.

En la memoria del proyecto arquitectónico queda patente la intención de respetar el edificio de Julio Bravo: El edificio a conservar queda totalmente representado y visible, unas veces desde fuera del nuevo edificio y otras formando parte del mismo (Pérez Latorre, 2006: 3). Sin embargo, el diálogo entre ambos componentes es complejo, ya que la volumetría de la ampliación resulta desproporcionada respecto a los antiguos talleres. La fachada de estos -aunque visible en algunas zonas- queda parcialmente escondida, de tal forma que es difícil percibir de forma clara sus características espaciales y entender el espacio original.

Junto con el edificio de los antiguos talleres Pignatelli, el anterior museo pasa también a segundo plano. Preguntado por cómo se había enfrentado al hecho de tener que sacrificar su anterior museo, Pérez Latorre afirmaba: $A$ veces, en la vida las cosas son así. Y para mejorar, hay que destruir'.

El anterior museo funciona como un gran basamento para la nueva obra, como la peana de una escultura. En palabras de Jesús Marco, también arquitecto, esta zona mantiene el zócalo histórico de la memoria del lugar ${ }^{10}$. Aunque se conserva la fachada de hormigón de la primera etapa del museo, la entrada

\footnotetext{
${ }^{7} \mathrm{El}$ arquitecto afirmaba: este edificio está realizado para ser visto en escorzo. Mariano García, “La ampliación del Museo Pablo Serrano estará acabada en octubre de este año", Heraldo de Aragón, (Zaragoza, 16-V-2010).

${ }^{8} \mathrm{Y}$ que pudieron ser contemplados en la exposición "Historia de un proyecto", una de las muestras inaugurales de la ampliación del IAACC Pablo Serrano.

${ }^{9}$ M. Navarcorena, "El museo Pablo Serrano abandera la arquitectura de vanguardia posExpo", El Periódico de Aragón, (Zaragoza, 15-XII-2008).

${ }^{10}$ J. Marco Llombart, "El nuevo rostro vertical de Zaragoza", Heraldo de Aragón, Suplemento Artes \& Letras, (Zaragoza, 28-IV-2011).
} 
principal ya no se realiza por el puente del que antes hablábamos (entrada que se mantiene para acceder al salón de actos o a la biblioteca) sino por el lado opuesto, por la intersección del paseo María Agustín con la calle Doctor Fleming.
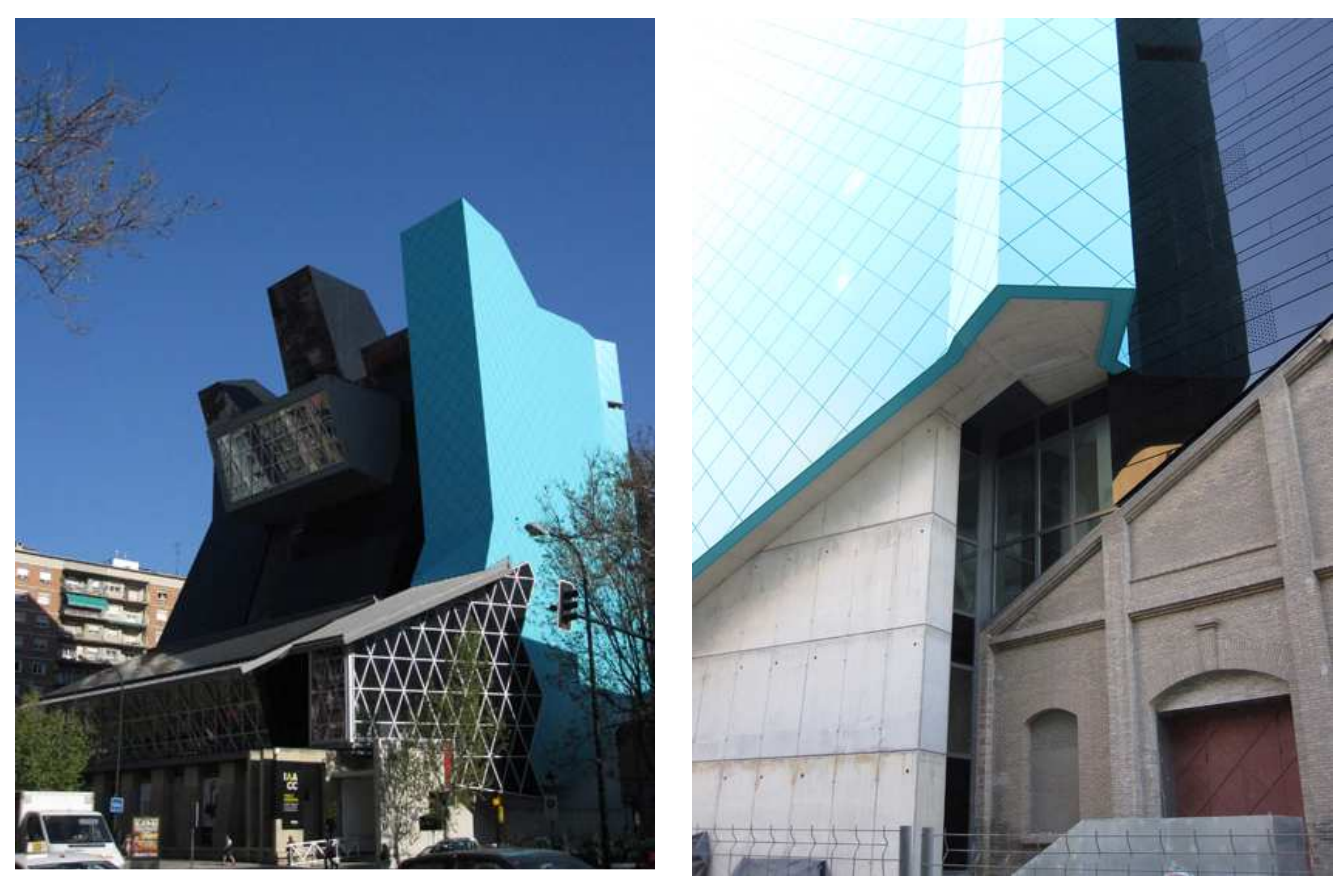

Figs. 7 - 8. Aspecto exterior y detalle de la fachada lateral del IAACC Pablo Serrano.

En cuanto a la inserción en la ciudad, Pérez Latorre proponía peatonalizar el tramo de la calle Doctor Fleming a la que da el museo, con el objeto de realzar el edificio y embellecer su entorno. Se buscaba crear un espacio peatonal ante la puerta del museo, un espacio amplio, a modo de antesala, para acceder y darle una mayor calidez al edificio ${ }^{11}$. Lo cierto es que la propuesta, que no se ha llevado a cabo, hubiera conseguido suavizar la zona adyacente a la entrada del IAACC y mejorar este paseo ciudadano.

${ }^{11}$ S. Campo, "El arquitecto Pérez Latorre plantea peatonalizar la calle de nuevo acceso al Pablo Serrano", Heraldo de Aragón, (Zaragoza, 17-XI-2010). 


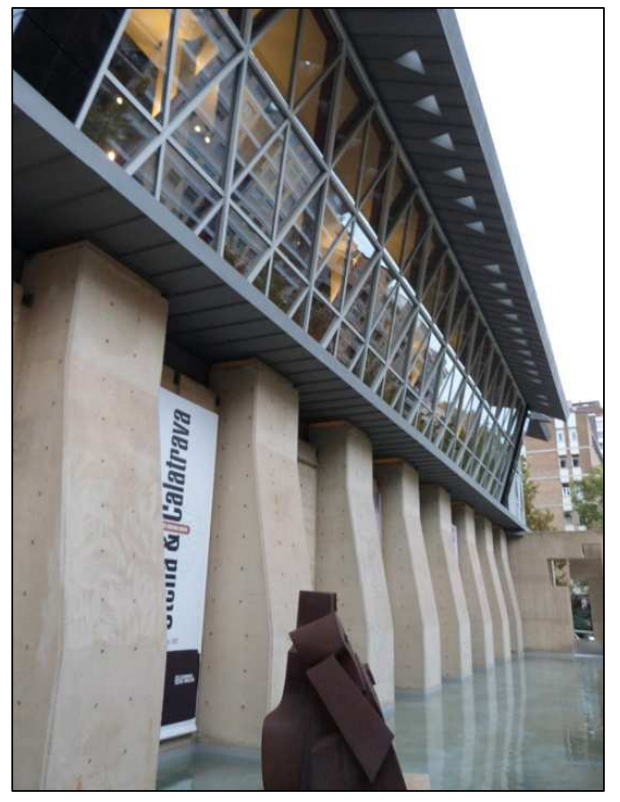

Fig. 9. Fachada actual del IAACC Pablo Serrano.

Aparte de las críticas por el desfase presupuestario en las obras (que tuvieron un sobrecoste de 7 millones de euros) o el alto coste de mantenimiento del edificio, el nuevo museo no dejó indiferente a casi nadie en la ciudad de Zaragoza, tanto en el ámbito cultural como entre los ciudadanos. Existió incluso una encuesta on-line puesta en marcha por el diario regional Heraldo de Aragón, que planteaba la pregunta "¿Le gusta la nueva fachada del museo?". La controversia suscitada en torno al edificio a medida que su aspecto externo se iba perfilando se reflejó en la prensa local: el museo recibía apelativos como "Transformer" o "monstruo"12, aunque también hubo quien lo definió como un "pequeño Guggenheim"13. La polémica no es algo nuevo para el arquitecto, ya que algunos de sus edificios, como el "cubo" del Museo del Foro de Caesaraugusta o el Auditorio de la ciudad han ocasionado tanto encendidas críticas como apasionadas alabanzas.

Entre los defensores del edificio se encuentran arquitectos como Jesús Marco, quien escribió el 28 de abril de 2011 en Heraldo de Aragón un artículo lleno de lirismo e imágenes poéticas (J. Marco, op.cit). Marco apuntaba con acierto una de las posibles razones por las que el edificio levantó tantas críticas en su momento: los habitantes de una ciudad se sienten molestos con la alteración de los paisajes urbanos a los que están acostumbrados. Marco define el nuevo museo como una tilde agitadora en un entrañable Paseo de nuestra ciudad, capaz de hacer girar la cabeza a un ciudadano más necesitado que nunca de ilusiones. También Antón Castro, periodista y escritor, rompía una lanza a fa-

${ }^{12}$ C.P.B., “Un edificio que no deja indiferente a nadie”, Heraldo de Aragón, (Zaragoza, 24-VII-2010).

${ }^{13}$ Ibídem. 
vor del edificio: Pérez Latorre ha creado una obra con personalidad, escultórica y arquitectónica a la vez, audaz, con énfasis de modernidad ${ }^{14}$. Sin embargo, Castro advertía de que el IAACC Pablo Serrano debía hacerse fuerte y necesario, y sorprendente en sus iniciativas y en su rigor, con una buena gestión cultural ${ }^{15}$.

Desde otros ámbitos de la cultura se escucharon opiniones menos favorables. El catedrático de Teoría de la Literatura de la Universidad de Zaragoza Luis Beltrán, en una mesa redonda sobre la situación del IAACC, se refería al nuevo edificio como un ejemplo de la "irrealidad" que estamos viviendo, un edificio "feo y extraño"16. El profesor Federico Torralba, preguntado en relación al museo, decía lo siguiente:

Está muy bien, estupendo en muchos aspectos, (...). Pero un edificio de esas dimensiones debe tener mucho 'movimiento' para que esté vivo. Lo lógico es que sea el museo de referencia de Aragón en cuanto a arte contemporáneo, porque tiene tres enormes salas para exposiciones temporales. Pero esa virtud es también un problema. No se puede llenar con muchos y pequeños elementos. Hay que organizar grandes exposiciones, y eso es caro. Es un museo con muchos problemas, a mi modo de ver ${ }^{17}$.

El paso del tiempo dirá si el museo se erige en un foco de modernidad, pero ello depende más del dinamismo que le impriman sus responsables que de su apariencia externa, la que sin duda acabará incorporándose a nuestra imagen ciudadana. Lo cierto es que el IAACC Pablo Serrano acoge ya todo tipo de eventos que van desde performances artísticas hasta presentaciones de libros, pasando por conciertos, conferencias, talleres, etc. Este dinamismo viene a paliar en cierta medida la debilidad inicial del centro en lo que se refiere a la política de exposiciones. Como decía Antón Castro en marzo de 2011, fecha de la reapertura del museo, Para abrir no se echa la casa por la ventana ${ }^{18}$. Las ex-

\footnotetext{
${ }^{14}$ Antón Castro, “Un viaje de doble dirección sin ira”, Heraldo de Aragón, (Zaragoza, 23-III-2011).

${ }^{15}$ Antón Castro, “Hoy podría empezar el futuro”, Heraldo de Aragón, (Zaragoza, 23-III-2011).

16 Soledad Campo, "El Museo Pablo Serrano corre el peligro de banalizarse”, Heraldo de Aragón, (Zaragoza, 1-XII-2011).

${ }^{17}$ Mariano García, “Federico Torralba: «Las galerías han llevado a la gente a comprar las firmas, no el arte»", Heraldo de Aragón, (Zaragoza, 15-V-2011).

18 Antón Castro, “Hoy podría empezar el futuro", Heraldo de Aragón, (Zaragoza, 23-III-2011).
} 
posiciones inaugurales fueron tres: la primera, “El IAACC Pablo Serrano, historia de un proyecto", dedicada a la propia gestación del edificio; "O con la estrella o en la cueva" (comisariada por Fernando Sinaga), una selección de la obra de Pablo Serrano; y la exposición colectiva "Noreste", de artistas aragoneses contemporáneos. Es decir, el nuevo IAACC abrió al público en marzo de 2011 sin una exposición mediática a la medida de la espectacularidad de su nueva arquitectura, que sirviera de reclamo para la afluencia de visitantes y que marcara un antes y un después en la historia cultural de la ciudad.

\section{Conclusiones.}

El análisis reposado de la historia constructiva del IAACC Pablo Serrano y del contexto urbano en el que se implanta permite valorar la iniciativa de forma adecuada, más allá de juicios espontáneos dictados por el aspecto externo del nuevo edificio.

Es innegable que la última intervención de José Manuel Pérez Latorre ha dado lugar a una arquitectura visualmente impactante, provocadora en sus formas, fruto de una libertad creativa (otorgada por los promotores del museo) llevada a sus máximas consecuencias. Resulta evidente que la arquitectura contemporánea puede (y debe) dejar su impronta en la ciudad, como ha sucedido en todas las épocas históricas, y que el arquitecto contemporáneo se encuentra ante el reto de proporcionar a la ciudad nuevas formas emblemáticas. No hay duda de que el IAACC Pablo Serrano es ahora una de ellas. Pero no es menos cierto que esta concepción de la arquitectura presenta el riesgo de crear ciudad a base de hitos urbanos que se dan la espalda los unos a los otros, que compiten en su individualidad y que no contribuyen a la definición de un tejido urbano trabado.

Desde el punto de vista de la recuperación del patrimonio industrial para usos museísticos, el nuevo IAACC Pablo Serrano formaría parte de esa primera tendencia más espectacular y radical en la rehabilitación de arquitectura industrial de la que hablaba Ascensión Hernández (2007: 32-35). El análisis de este ejemplo permite constatar la pérdida parcial de la idiosincrasia del edificio original, que queda reducido en la última intervención a un conjunto de fachadas integradas a la fuerza en la nueva obra y ciertas reminiscencias a la cubierta original en la sala de exposiciones de la planta baja. Las proporciones 
del edificio industrial de los talleres de Julio Bravo resultan difícilmente perceptibles, perdiéndose de esta forma la seña de identidad del espacio.

Dicho esto, hay que tener en cuenta la complejidad de construir en un entorno como el que hemos tenido ocasión de analizar. Entendemos que el carácter inconexo de la urbanización, a base de hitos aislados y no siempre bien resueltos, plantea un punto de partida poco sugerente. Quizá el no tener en cuenta en absoluto este contexto sea una posible solución. Por otro lado, la arquitectura contemporánea no debería perder un cierto carácter provocador, del que sin duda el arquitecto se ha servido en esta obra. Una nueva presencia que provoque al ciudadano, que le haga reaccionar, le invite a interrogarse sobre la ciudad y le haga ser consciente del escenario en el que vive, ya sea para felicitarse por la nueva imagen ciudadana o para indignarse por su presencia.

\section{Bibliografía.}

ANÓNIMO (1987): “Fundación Pablo Serrano. Arquitecto: José Manuel Pérez Latorre", Aldaba, № 7, Colegio Oficial de Arquitectos de Aragón, Zaragoza, págs. 28-31.

Ansón Navarro, A. (2007): El entorno del Convento del Carmen de Zaragoza. Una reconstrucción histórica y artística. Siglos XIII al XX, Elazar, Zaragoza.

HERNÁNDEZ MARTÍNEZ, A. (2006): “La musealización de la arquitectura industrial. Algunos casos de estudio". En RIVERA, J. (ed.). AREPA Actas del IV Congreso Internacional "Restaurar la memoria" Arqueología, Arte y Restauración (Valladolid, 2004), Junta de Castilla y León, Valladolid, págs. 533-556.

LORENTE LORENTE, J. P. (1999): “Vino nuevo en viejas cubas: artistas, galeristas y museos / centros de arte contemporáneo en antiguas naves industriales", Artigrama, № 14, Universidad de Zaragoza, Departamento de Historia del Arte, Zaragoza, págs. 183-204.

MArtínez MolinA, J. (2009): “Nuevas aportaciones al estudio de la Puerta del Carmen de Zaragoza (1787-1795)", Artigrama, № 24, Universidad de Zaragoza, Departamento de Historia del Arte, Zaragoza, págs. 443-466.

Pérez Latorre, J. M. (1990): Proyecto refundido "Fundación Pablo Serrano". (Inédito).

PÉRez LATORRe, J. M. (2006): Proyecto de ejecución. Memoria y ampliación del Museo Pablo Serrano. (Inédito). 